\title{
Observation of a Class of Disturbance in Time Series Expansion for Fractional Order Systems
}

\author{
Yiheng Wei, ${ }^{1}$ Hamid Reza Karimi, ${ }^{2}$ Jinwen Pan, ${ }^{1}$ Qing Gao, ${ }^{1,3}$ and Yong Wang \\ ${ }^{1}$ Department of Automation, University of Science and Technology of China, Hefei 230027, China \\ ${ }^{2}$ Department of Engineering, Faculty of Engineering and Science, University of Agder, 4898 Grimstad, Norway \\ ${ }^{3}$ Department of Mechanical and Biomedical Engineering, City University of Hong Kong, Hong Kong
}

Correspondence should be addressed to Yiheng Wei; neudawei@mail.ustc.edu.cn

Received 24 December 2013; Accepted 2 February 2014; Published 17 March 2014

Academic Editor: Jun $\mathrm{Hu}$

Copyright (C) 2014 Yiheng Wei et al. This is an open access article distributed under the Creative Commons Attribution License, which permits unrestricted use, distribution, and reproduction in any medium, provided the original work is properly cited.

\begin{abstract}
This paper is concerned with the problem of designing disturbance observer for fractional order systems, of which the disturbance is in time series expansion. The stability of a special observer with the selected nonlinear weighted function and transient dynamics function is rigorously analyzed for slowly varying disturbance. In addition, the result is also extended to estimate slope forms disturbance and higher order disturbance of fractional order systems. The efficacy of the proposed method is validated through numerical examples.
\end{abstract}

\section{Introduction}

In recent years, fractional order systems (FOSs) have attracted considerable attention from control community, since many engineering plants and processes cannot be described concisely and precisely without the introduction of fractional order calculus [1-4]. Due to the tremendous efforts devoted by researchers, a number of valuable results on stability analysis [5-7] and controller synthesis [8-10] of FOSs have been reported in the literature.

Like the integer order system, the disturbance always exists in the FOSs and usually it is not possible or practical to obtain the exact model of the FOSs, so disturbance or uncertainty observation has been one of the major issues in the control field. A rich body of results about disturbance observation have been reported in the literature with different methods. For example, the so-called Q-filter method estimates the disturbance depending on the inversion of the transfer function. Many applications based on Q-filter have been reported in process control fields [11-13]. The second class mainly refers to the active disturbance rejection control technique proposed by Han [14]. Under this framework, the extended state observer can estimate the disturbance effectively, which has been demonstrated in many fields [1517]. The third class, called recursive filtering methods, is from a statistical point of view [18-20]. Some other methods about disturbance observation have been developed in [21-25].

A special kind of disturbance in time series expansion is considered to solve the friction compensation problem in [26]. The algorithms are brief and effective. Additionally, the time series expansion form is the basic form of the nonstochastic disturbance in engineering. Intuitively, such useful methods need to be generalized to FOSs. Maybe the stability is hard to be prove; such disturbance observation for FOSs has not been introduced so far in the literature to the best of the authors' knowledge. The proposed approaches in [26] reveal several important aspects: (1) the linear observer converges in the form of exponent, which means that it will take the observance error infinity time to converge to zero; (2) the observance value may bring forth big overshoot when the disturbance is discontinuous; meanwhile, the possible conflict between the overshoot and rapidity always exists; and (3) the disturbance is not general enough, since its degrees are restricted to integers. Motivated by the reasons stated above, in this paper we focus on the observation of a class of disturbance in time series expansion for FOSs. 
The following section is devoted to some basic background materials and the main problems. Discussing the more general disturbance using a special nonlinear function, the main results are presented in Section 3. In Section 4, some numerical examples are provided to illustrate the effectiveness and advantages of the proposed approach. Conclusions are given in Section 5 .

\section{Problem Formulation and Preliminaries}

Consider the following fractional order system:

$$
\mathscr{D}^{\alpha} x=f(x, u ; t)+F d(t),
$$

where the system order $0<\alpha<1 . x \in \mathbb{R}^{n}, u \in \mathbb{R}^{m}$, and $d \in \mathbb{R}^{r}$ are the system state, the control input, and the unknown disturbance, respectively. $f(\cdot)$ and the matrix $F$ with $\operatorname{rank}(F)=r$ are known. It is assumed that the state variable $x$ is measured and the initial state condition $x(0)$ is known.

The reduced-order system can be expressed as

$$
F^{+} \mathscr{D}^{\alpha} x=F^{+} f(x, u ; t)+d(t)
$$

where $F^{+}=\left(F^{T} F\right)^{-1} F^{T}$ is the Moore-Penrose pseudoinverse of $F$.

Consider the disturbance has the following form:

$$
d(t)=\sum_{i=0}^{k} d_{i} t^{n_{i}}
$$

where $d_{i}(i \in \mathscr{K} \triangleq\{0,1, \ldots, k\})$ is constant but unknown and $n_{i}<n_{i+1}(i \in \mathscr{K}-\{k\})$ holds. Due to the magnitude of $n_{k}$, the disturbance $d(t)$ can be divided into the following three categories:

(i) slowly varying disturbance $n_{k}<\alpha$;

(ii) slope forms disturbance $\alpha \leq n_{k}<2 \alpha$;

(iii) higher order disturbance $n_{k} \geq 2 \alpha$.

The following Caputo definition [1] is adopted for fractional derivatives of order $\alpha$ for function $f(t)$ :

$$
\mathscr{D}^{\alpha} f(t)=\frac{1}{\Gamma(n-\alpha)} \int_{0}^{t}(t-\tau)^{n-\alpha-1} f^{(n)}(\tau) \mathrm{d} \tau,
$$

where the fractional order $n-1<\alpha \leq n, n \in \mathbb{N}$, and the Gamma function $\Gamma(x)=\int_{0}^{\infty} e^{-t} t^{x-1} \mathrm{~d} t$.

The so-called fractional order integral is just the dual operation of the fractional order differential. If $v(t)$ is the $\alpha$ th order differential of $y(t)$, then

$$
\begin{aligned}
& v(t)=\mathscr{D}^{\alpha}(y(t)), \\
& y(t)=\mathscr{I}_{\alpha}(v(t)) .
\end{aligned}
$$

In order to describe briefly later, we introduce the following definition.
Definition 1. Consider polynomials $\varphi(s)$ with commensurate order $\alpha$

$$
\varphi(s)=s^{n \alpha}+a_{0} s^{(n-1) \alpha}+\cdots+a_{n-2} s^{\alpha}+a_{n-1},
$$

and define the corresponding characteristic matrix $\mathscr{A}$ as

$$
\mathscr{A}=\left[\begin{array}{ccccc}
-a_{0} & -a_{1} & \cdots & -a_{n-2} & -a_{n-1} \\
1 & 0 & \cdots & 0 & 0 \\
0 & 1 & \cdots & 0 & 0 \\
\vdots & \vdots & \ddots & 0 & 0 \\
0 & 0 & \cdots & 1 & 0
\end{array}\right]
$$

$\varphi(s)$ in (6) is said to be stable if all the eigenvalues of $\mathscr{A}$ are in the region $\mathscr{D}(\alpha) \triangleq\{s \in \mathbb{C}:|\arg (s)|>\alpha \pi / 2\}$.

\section{Main Results}

\subsection{Slowly Varying Disturbance Observer}

Theorem 2 (FDOB0). Given a constant matrix $\Lambda_{0}=$ $\operatorname{diag}\left(\lambda_{01}, \lambda_{02}, \ldots, \lambda_{0 r}\right)$, where $\lambda_{0 i}>0, i \in \mathscr{R} \triangleq\{1, \ldots, r\}$, the disturbance observer given by

$$
\begin{gathered}
\widehat{d}=\Lambda_{0} g_{0}(e), \\
\mathscr{D}^{\alpha} z=F^{+} f(x, u ; t)+\frac{\widehat{d}}{t s(t)}
\end{gathered}
$$

is asymptotically convergent to the slowly varying disturbance when the nonlinear weighted function

$$
g_{0}(e)=\left[g_{01}\left(e_{1}\right) g_{02}\left(e_{2}\right) \cdots g_{0 r}\left(e_{r}\right)\right]^{T}
$$

is selected as

$$
g_{0 i}\left(e_{i}\right)= \begin{cases}\left|\frac{e_{i}}{w}\right|^{\sigma} \operatorname{sgn}\left(\frac{e_{i}}{w}\right), & \left|e_{i}\right| \geq w \\ \frac{e_{i}}{w}, & \left|e_{i}\right|<w\end{cases}
$$

where $e \triangleq F^{+} x-z=\left[e_{1} e_{2} \cdots e_{r}\right]^{T}, z \in \mathbb{R}^{r}, z(0)=F^{+} x(0)$, $\sigma>0, w>0$ for any $i \in \mathscr{R}$, and the transient dynamics function $t s(t)$ is given by

$$
t s(t)= \begin{cases}0.51+0.49 \sin \left(\frac{\pi t}{T_{0}}-\frac{\pi}{2}\right), & t \leq T_{0} \\ 1, & t>T_{0} .\end{cases}
$$

Proof. Defining the observation error $\varepsilon=d-\widehat{d} / t s(t)$ yields

$$
\mathscr{D}^{\alpha} \varepsilon=\mathscr{D}^{\alpha} d-\mathscr{D}^{\alpha}\left(\frac{\widehat{d}}{t s(t)}\right) \text {. }
$$


Based on the Leibniz rule for fractional differentiation, one has

$$
\begin{aligned}
\mathscr{D}^{\alpha} & \left(\frac{\hat{d}}{t s(t)}\right) \\
& =\sum_{i=0}^{\infty}\left(\begin{array}{c}
\alpha \\
i
\end{array}\right)\left(\frac{1}{t s(t)}\right)^{(i)} \mathscr{D}^{\alpha-i} \hat{d} \\
& =\frac{1}{t s(t)} \mathscr{D}^{\alpha} \hat{d}+\sum_{i=1}^{\infty}\left(\begin{array}{c}
\alpha \\
i
\end{array}\right)\left(\frac{1}{t s(t)}\right)^{(i)} \mathscr{D}^{\alpha-i} \hat{d} \\
& =\frac{1}{t s(t)} \mathscr{D}^{\alpha} \hat{d}+h(t),
\end{aligned}
$$

where the Newtons binomial generalized to noninteger orders as $\left(\begin{array}{c}\alpha \\ i\end{array}\right)=\Gamma(\alpha+1) / \Gamma(\alpha-i+1) \Gamma(i+1)$.

Substituting (13) into (12) and linearizing the system at $\bar{e}$, one can get

$$
\begin{aligned}
\mathscr{D}^{\alpha} \mathcal{\varepsilon} & =\mathscr{D}^{\alpha} d-\frac{1}{t s(t)} \Lambda_{0} \mathscr{D}^{\alpha} g_{0}(e)-h(t) \\
& =\mathscr{D}^{\alpha} d-\left.\frac{1}{t s(t)} \Lambda_{0} J\right|_{e=\bar{e}} \mathscr{D}^{\alpha} g_{0}(e)-h(t) \\
& =\mathscr{D}^{\alpha} d-\left.\frac{1}{t s(t)} \Lambda_{0} J\right|_{e=\bar{e}} \varepsilon-h(t),
\end{aligned}
$$

where $J$ is the pseudo-Jacobi matrix of $g_{0}(e)$ with

$$
J=\left[\begin{array}{ccc}
\frac{\partial g_{01}}{\partial e_{1}} & \cdots & \frac{\partial g_{01}}{\partial e_{r}} \\
\vdots & \ddots & \vdots \\
\frac{\partial g_{0 r}}{\partial e_{1}} & \cdots & \frac{\partial g_{0 r}}{\partial e_{r}}
\end{array}\right]=\left[\begin{array}{ccc}
\frac{\mathrm{d} g_{01}}{\mathrm{~d} e_{1}} & \cdots & 0 \\
\vdots & \ddots & \vdots \\
0 & \cdots & \frac{\mathrm{d} g_{0 r}}{\mathrm{~d} e_{r}}
\end{array}\right]
$$

Define a new variable $\mu \triangleq 1 / t s(t)$; then one has $\mu \in[1,50]$ and the time-varying system in (14) can be regarded as a timeinvariant system (16) with the interval uncertain parameter $\mu$ :

$$
\mathscr{D}^{\alpha} \mathcal{\varepsilon}=\mathscr{D}^{\alpha} d-h(t)-\left.\mu \Lambda_{0} J\right|_{e=\bar{e}} \varepsilon .
$$

From (10), for all $i \in \mathscr{R}$, one can easily get

$$
\frac{\mathrm{d} g_{0 i}\left(e_{i}\right)}{\mathrm{d} e_{i}}= \begin{cases}\frac{\sigma}{w}\left|\frac{e_{i}}{w}\right|^{\sigma-1}, & \left|e_{i}\right| \geq w, \\ \frac{1}{w}, & \left|e_{i}\right|<w .\end{cases}
$$

Note that $\sigma>0$ and $w>0$, so the following result establishes:

$$
\frac{\mathrm{d} g_{0 i}\left(e_{i}\right)}{\mathrm{d} e_{i}} \in \begin{cases}\left(0, \frac{1}{w}\right], & 0<\sigma \leq 1, \\ {\left[\frac{1}{w}, \infty\right),} & \sigma>1 .\end{cases}
$$

Thus, the matrix inequality $\left.J\right|_{e=\bar{e}}>0$ always holds.

From the Caputo definition of fractional derivatives, one has

$$
\mathscr{D}^{\alpha} d=\sum_{i=0}^{k} \frac{d_{i} \Gamma\left(n_{i}+1\right)}{\Gamma\left(n_{i}+1-\alpha\right)} t^{n_{i}-\alpha} .
$$

Due to (8) and $n_{k}<\alpha$, one obtains that the first term of the right-hand side of (16) vanishes as $t \rightarrow \infty$ and the second term is equal to 0 when $t>T_{0}$. No matter $\mu=1$ or $\mu=50$, the error dynamic system (16) is stable, so (16) is stable for all the admissible uncertainties and the final value of observation error exists.

The Laplace transform of (16) is

$$
\begin{aligned}
& s^{\alpha} E(s)-s^{\alpha-1} \mathcal{E}(0) \\
& \quad=s^{\alpha} D(s)-s^{\alpha-1} d(0)-\left.\Lambda_{0} J\right|_{e=\bar{e}} E(s)-H(s),
\end{aligned}
$$

where $E(s), D(s)$, and $H(s)$ are the Laplace transforms of $\varepsilon(t), d(t)$, and $h(t)$, respectively.

According to the initial condition, one has that

$$
\widehat{d}(0)=\Lambda_{0} g_{0}\left(F^{+} x(0)-z(0)\right)=\Lambda_{0} g_{0}(0)=0 .
$$

Consequently, the following equation holds:

$$
\varepsilon(0)=d(0)-\widehat{d}(0)=d(0) .
$$

Define $k_{i}=\left.\mu\left(\mathrm{d} g_{0 i}\left(e_{i}\right) / \mathrm{d} e_{i}\right)\right|_{e_{i}=\bar{e}_{i}}, i \in \mathscr{R}$, and consider $\Lambda_{0}$ and $\left.J\right|_{e=\bar{e}}$ are all diagonal; one gets the decoupled error dynamics from (19):

$$
s^{\alpha} E_{i}(s)+\lambda_{0 i} k_{i} E_{i}(s)=s^{\alpha} D_{i}(s)-H_{i}(s), \quad i \in \mathscr{R} .
$$

For the slowly varying disturbance, one has $\alpha-n_{k}>0$ and finite positive constant $\lambda_{0 i} k_{i}$. Then using the Final-Value Theorem for any $i \in \mathscr{R}$, one obtains

$$
\begin{aligned}
e_{i}(\infty) & =\lim _{s \rightarrow 0} s E_{i}(s) \\
& =\lim _{s \rightarrow 0} s \frac{s^{\alpha} D_{i}(s)-H_{i}(s)}{s^{\alpha}+\lambda_{0 i} k_{i}} \\
& =\lim _{s \rightarrow 0} s \frac{s^{\alpha} \sum_{i=0}^{k}\left(d_{i} \Gamma\left(n_{i}+1\right) / s^{n_{i}+1}\right)-H_{i}(s)}{s^{\alpha}+\lambda_{0 i} k_{i}}=0 .
\end{aligned}
$$

Therefore, the observation error is asymptotically stable and the initial error depends on the time derivative of the disturbance for any $\bar{e}$. In other words, the disturbance observer would exactly estimate the disturbance in the steady state. The proof is completed.

Remark 3. Regarding stability, any of the matrices whose eigenvalues are all in the region $\mathscr{D}(\alpha)$ can be adopted for $\Lambda_{0}$. In order to have the decoupled error dynamics, we consider a class of diagonal matrices for $\Lambda_{0}$ and the nonlinear weighted function $g_{0}(e)$ consists of $g_{0 i}\left(e_{i}\right)$ rather than $g_{0 i}(e), i \in \mathscr{R}$.

Remark 4. The functions $g_{0}(e)$ and $t s(t)$ in (8) are not unique. Considering only the stability, any nonlinear or linear function with positive derivative can be adopted for $g_{0}(e)$, and any nonlinear or linear function with positive value capable of arranging the transition dynamic can be adopted for $t s(t)$. However, without loss of generality, two classes of nonlinear functions with the forms (10) and (11) are considered. 
Remark 5. Considering $\sigma=1, w=1, T_{0}=1$, and $g_{0}(e)=$ $F^{+} x-z$, then FDOB0 will reduce to linear disturbance observer which are right a set of low-pass filters $\lambda_{0 i} /\left(s^{\alpha}+\lambda_{0 i}\right)$, $i \in \mathscr{R}$. Actually, DOB0 in [26] can be viewed as a specific case of such linear FDOB0 with $\alpha=1$.

3.2. Slope Forms Disturbance Observer. Consider the slope forms disturbance; Theorem 2 is extended to Theorem 6 as follows.

Theorem 6 (FDOB1). Given two constant matrices $\Lambda_{0}=$ $\operatorname{diag}\left(\lambda_{01}, \lambda_{02}, \ldots, \lambda_{0 r}\right)$ and $\Lambda_{1}=\operatorname{diag}\left(\lambda_{11}, \lambda_{12}, \ldots, \lambda_{1 r}\right)$, suppose that $\lambda_{i j}(i \in\{0,1\}, j \in \mathscr{R})$ is chosen such that the polynomial $\varphi_{j}(s)=s^{2 \alpha}+\lambda_{0 j} s^{\alpha}+\lambda_{1 j}$ is stable; then the disturbance observer given by

$$
\begin{gathered}
\widehat{d}=\Lambda_{0} g_{0}(e)+\Lambda_{1} \mathscr{I}_{\alpha}\left(g_{0}(e)\right), \\
\mathscr{D}^{\alpha} z=F^{+} f(x, u ; t)+\frac{\widehat{d}}{t s(t)}
\end{gathered}
$$

is asymptotically convergent to the slope forms disturbance when the initial nonlinear weighted function $g_{0}(e)$ has the same form as Theorem 2 and the coefficients $\sigma, w$, and $T_{0}$ are properly selected according to $\Lambda_{0}$ and $\Lambda_{1}$.

Proof. Consider the same observation error $\varepsilon$ with Theorem 2 and linearize the system at $\bar{e}$; one can get

$$
\begin{aligned}
\mathscr{D}^{2 \alpha} \varepsilon & =\mathscr{D}^{2 \alpha} d-\mathscr{D}^{2 \alpha} \hat{d} \\
& =\mathscr{D}^{2 \alpha} d-\mu \Lambda_{0} \mathscr{D}^{2 \alpha} g_{0}(e)-\mu \Lambda_{1} \mathscr{D}^{\alpha} g_{0}(e)-h(t) \\
& =\mathscr{D}^{2 \alpha} d-\left.\mu \Lambda_{0} J\right|_{e=\bar{e}} \mathscr{D}^{2 \alpha} e-\left.\mu \Lambda_{1} J\right|_{e=\bar{e}} \mathscr{D}^{\alpha} e-h(t) \\
& =\mathscr{D}^{2 \alpha} d-\left.\mu \Lambda_{0} J\right|_{e=\bar{e}} \mathscr{D}^{\alpha} \varepsilon-\left.\mu \Lambda_{1} J\right|_{e=\bar{e} \varepsilon}-h(t),
\end{aligned}
$$

where $J$ is defined the same with Theorem 2 and $h(t)$ is equal to 0 when $t>T_{0}$, which is given by

$$
h(t)=\sum_{i=1}^{\infty}\left(\begin{array}{c}
2 \alpha \\
i
\end{array}\right) \mu^{(i)} \mathscr{D}^{2 \alpha-i} \widehat{d} .
$$

Because $\Lambda_{0}$ and $\Lambda_{1}$ are diagonal matrices and $\left.J\right|_{e=\bar{e}}$ is a positive definite matrix, one has the decoupled error dynamics. Therefore, the problem becomes that given stable polynomial $\varphi_{i}(s)=s^{2 \alpha}+\lambda_{0 i} s^{\alpha}+\lambda_{1 i}, i \in \mathscr{R}$, properly select $\sigma, w$ and $T_{0}$ to keep the polynomial $\bar{\varphi}_{i}(s)=s^{2 \alpha}+\lambda_{0 i} k_{i} s^{\alpha}+\lambda_{1 i} k_{i}$ is always stable for all admissible uncertainties.

Set $\gamma=s^{\alpha}$; then the characteristic equations $\varphi_{i}(s)=0$ and $\bar{\varphi}_{i}(s)=0$ can be rewritten as (28) and (29), respectively:

$$
\begin{gathered}
\gamma^{2}+\lambda_{0 i} \gamma+\lambda_{1 i}=0, \\
\gamma^{2}+\lambda_{0 i} k_{i} \gamma+\lambda_{1 i} k_{i}=0 .
\end{gathered}
$$

Suppose $\gamma_{1}$ and $\gamma_{2}$ are the roots of (28); then the following discussion will be divided into two cases.
(A) $\gamma_{1}$ and $\gamma_{2}$ Are Nonconjugated Roots. Without loss of generality, we set $\gamma_{1}=a$ and $\gamma_{2}=b$ with $a \leq b<0$. Then the characteristic equation of (29) can be described as

$$
\gamma^{2}-(a+b) k_{i} \gamma+a b k_{i}=0
$$

Calculating the roots of the quadratic equation yields

$$
\gamma_{3}=\frac{(a+b) k_{i}+\sqrt{\Delta}}{2}, \quad \gamma_{4}=\frac{(a+b) k_{i}-\sqrt{\Delta}}{2},
$$

where the discriminant $\Delta=(a+b)^{2} k_{i}^{2}-4 a b k_{i}$.

When $k_{i} \geq 4 a b /(a+b)^{2}$, then $\Delta \geq 0$ and moreover

$$
\gamma_{4} \leq \gamma_{3}<0
$$

Thus $\bar{\varphi}_{i}(s)$ is stable.

When $k_{i}<4 a b /(a+b)^{2}$, then $\Delta<0$ and moreover

$$
\left|\arg \left(\gamma_{3}\right)\right|=\left|\arg \left(\gamma_{4}\right)\right|>\frac{\pi}{2} \geq \frac{\alpha \pi}{2} .
$$

Thus $\bar{\varphi}_{i}(s)$ is stable.

Overall, when $\varphi_{i}(s)$ has two nonconjugated roots, as long as $\sigma$ and $w$ are positive, $\bar{\varphi}_{i}(s)$ is stable $(i \in \mathscr{R})$.

(B) $\gamma_{1}$ and $\gamma_{2}$ Are Conjugated Roots. Let $\gamma_{1}=c+d i$ and $\gamma_{2}=$ $c-d i$ with $c, d \in \mathbb{R},\left|\arg \left(\gamma_{1}\right)\right|>\alpha \pi / 2$. Then the characteristic equation of (25) can be described as

$$
\gamma^{2}-2 c k_{i} \gamma+\left(c^{2}+d^{2}\right) k_{i}=0
$$

Calculating the roots of the quadratic equation yields

$$
\gamma_{3}=\frac{2 c k_{i}+\sqrt{\Delta}}{2}, \quad \gamma_{4}=\frac{2 c k_{i}-\sqrt{\Delta}}{2}
$$

where the discriminant $\Delta=4 k_{i}^{2} c^{2}-4 k_{i}\left(c^{2}+d^{2}\right)$.

When $k_{i} \geq\left(c^{2}+d^{2}\right) / c^{2}, \Delta \geq 0, \gamma_{3}$ and $\gamma_{4}$ are real roots. If $c<0$, one gets

$$
\gamma_{4} \leq \gamma_{3}<0
$$

Thus $\bar{\varphi}_{i}(s)$ is stable.

If $c \geq 0$, one gets

$$
0<\gamma_{4} \leq \gamma_{3}
$$

Thus $\bar{\varphi}_{i}(s)$ is unstable.

When $k_{i}<\left(c^{2}+d^{2}\right) / c^{2}, \Delta<0, \gamma_{3}$ and $\gamma_{4}$ are conjugated roots. If $c<0$, one gets

$$
\left|\arg \left(\gamma_{3}\right)\right|=\left|\arg \left(\gamma_{4}\right)\right|>\frac{\pi}{2} \geq \frac{\alpha \pi}{2} .
$$

Thus $\bar{\varphi}_{i}(s)$ is stable.

If $c \geq 0,\left|\arg \left(\gamma_{3}\right)\right|=\left|\arg \left(\gamma_{4}\right)\right|>\alpha \pi / 2$ is equivalent to the following:

$$
\tan \left(\left|\arg \left(\gamma_{3}\right)\right|\right)=\tan \left(\left|\arg \left(\gamma_{4}\right)\right|\right)=\left|\frac{\sqrt{-\Delta}}{2 k_{i} c}\right|>\tan \left(\frac{\alpha \pi}{2}\right) .
$$

Thus one obtains that if $k_{i}<\left(c^{2}+d^{2}\right)^{2} / c^{2}\left[1+\tan ^{2}(\alpha \pi / 2)\right]$, $\bar{\varphi}_{i}(s)$ is stable.

To sum up the above arguments, if $\varphi_{i}(s)$ has two conjugated roots, one gets the following results. 
(i) When $c<0, \bar{\varphi}_{i}(s)(i \in \mathscr{R})$ is always stable with $\sigma>0$, $w>0$.

(ii) When $c \geq 0$, both $\sigma<1, w<50 c^{2}\left[1+\tan ^{2}(\alpha \pi /\right.$ $2)] /\left(c^{2}+d^{2}\right)^{2}$ and $\sigma \geq 1, w<c^{2} /\left(c^{2}+d^{2}\right)^{2}$ can make sure $\bar{\varphi}_{i}(s)(i \in \mathscr{R})$ is stable.

Consequently, selecting $\sigma, w$ properly, all the error dynamic systems (26) are stable and the final value of observation errors exist.

When $0<2 \alpha \leq 1$, using the Laplace transform to (26), one has

$$
\begin{aligned}
s^{2 \alpha} E(s)-s^{2 \alpha-1} \varepsilon(0) \\
=s^{2 \alpha} D(s)-s^{2 \alpha-1} d(0)-\left.s^{\alpha} \mu \Lambda_{0} J\right|_{e=\bar{e}} E(s) \\
\quad+s^{\alpha-1} \varepsilon(0)-\left.\mu \Lambda_{1} J\right|_{e=\bar{e}} E(s)-H(s) .
\end{aligned}
$$

According to Theorem 2, one knows $e(0)=d(0)$. Therefore, (40) can be simplified as

$$
\begin{gathered}
s^{2 \alpha} E(s)+\left.s^{\alpha} \mu \Lambda_{0} J\right|_{e=\bar{e}} E(s)+\left.\mu \Lambda_{1} J\right|_{e=\bar{e}} E(s) \\
=s^{2 \alpha} D(s)+s^{\alpha-1} \varepsilon(0) H(s) .
\end{gathered}
$$

By using the Final-Value Theorem for any $i \in \mathscr{R}$, it follows that

$$
\begin{aligned}
\varepsilon_{i}(\infty) & =\lim _{s \rightarrow 0} s E_{i}(s) \\
& =\lim _{s \rightarrow 0} s \frac{s^{2 \alpha} D_{i}(s)+s^{\alpha-1} \varepsilon_{i}(0)}{s^{2 \alpha}+k_{i} \lambda_{0 i} s^{\alpha}+k_{i} \lambda_{1 i}}=0 .
\end{aligned}
$$

When $2 \alpha>1$, using the Laplace transform to (26), one has

$$
\begin{aligned}
s^{2 \alpha} E(s)-s^{2 \alpha-1} \varepsilon(0)-s^{2 \alpha-2} \dot{\varepsilon}(0) \\
=s^{2 \alpha} D(s)-s^{2 \alpha-1} d(0)-s^{2 \alpha-2} \dot{d}(0) \\
-\left.s^{\alpha} \mu \Lambda_{0} J\right|_{e=\bar{e}} E(s)+s^{\alpha-1} \varepsilon(0) \\
-\left.\mu \Lambda_{1} J\right|_{e=\bar{e}} E(s)-H(s) .
\end{aligned}
$$

Proceeding forward, we have $e(0)=d(0)$. Based on the FinalValue Theorem for any $i \in \mathscr{R}$, one has

$$
\begin{aligned}
& \mathcal{E}_{i}(\infty) \\
& =\lim _{x \rightarrow 0} s E_{i}(s) \\
& =\lim _{x \rightarrow 0} s \frac{s^{2 \alpha} D_{i}(s)+s^{\alpha-1} \varepsilon_{i}(0)+s^{2 \alpha-2} \dot{\varepsilon}_{i}(0)-s^{2 \alpha-2} \dot{d}_{i}(0)}{s^{2 \alpha}+k_{i} \lambda_{0 i} s^{\alpha}+k_{i} \lambda_{1 i}} \\
& =0 .
\end{aligned}
$$

Therefore, for any $\bar{e}$ the observation error is asymptotically stable and convergent to zero. To sum up, Theorem 6 has been completely proved.
Remark 7. Also considering that $\sigma=1, w=1, T_{0}=0$, and $g_{0}(e)=F^{+} x-z$, then FODB1 can reduce to linear disturbance observer, which actually consists of a set of low-pass filters $\left(\lambda_{0 i} s^{\alpha}+\lambda_{1 i}\right) /\left(s^{2 \alpha}+\lambda_{0 i} s^{\alpha}+\lambda_{1 i}\right), i \in \mathscr{R}$. Set $\alpha$ further; FDOB1 results in DOB1 in [26].

3.3. Higher Order Disturbance Observer. According to the understanding of the aforementioned approaches, one generalizes Theorem 2 further to observe the higher order disturbance.

Theorem 8 (FDOBp). Given constant matrices $\Lambda_{i}=\operatorname{diag}\left(\lambda_{i 1}\right.$, $\left.\lambda_{i 2}, \ldots, \lambda_{i r}\right)$ and nonlinear weighted functions $g_{i}(e)=$ $\mathscr{I}_{i \alpha}\left(g_{0}(e)\right), i \in \mathscr{P} \triangleq\{0,1, \ldots, p\}$, where $\lambda_{i j}(j \in \mathscr{R})$ is chosen from the stable polynomial $\varphi_{j}(s)=s^{(p+1) \alpha}+\sum_{i=0}^{p} \lambda_{i j} s^{(p-i) \alpha}$, then the disturbance observer given by

$$
\begin{gathered}
\widehat{d}=\sum_{i=0}^{p} \Lambda_{i} g_{i}(e), \\
\mathscr{D}^{\alpha} z=F^{+} f(x, u ; t)+\frac{\widehat{d}}{t s(t)}
\end{gathered}
$$

is asymptotically convergent to the higher order disturbance with $p \alpha \leq n_{k}<(p+1) \alpha$ when $g_{0}(e)$ has the same form as Theorem 2 and the coefficients $\sigma, w$, and $T_{0}$ are properly selected according to $\Lambda_{i}, i \in \mathscr{P}$.

Remark 9. The theorem can be generalized by using the similar aforementioned approach; wherefore the proof is omitted here.

Remark 10. Set $\sigma=1, w=1, T_{0}=0$, and $g_{0}(e)=F^{+} x-z$; then FDOBp becomes a linear disturbance observer, which is really a set of low-pass filters $\left(\lambda_{0 i} s^{p \alpha}+\cdots+\lambda_{(p-1) i} s+\right.$ $\left.\lambda_{p i}\right) /\left(s^{(p+1) \alpha}+\cdots+\lambda_{(p-1) i} s+\lambda_{p i}\right), i \in \mathscr{R}$. Furthermore, set $\alpha=1$; then FODBp will reduce to the higher order in [26].

Remark 11. Under certain conditions, all the above mentioned theorems (Theorems 2, 6, and 8) can be extended to the case $1<\alpha<2$.

Remark 12. The proposed method has a great design freedom. With the appropriate parameters, the FDOBp is a fast nonovershooting disturbance observer for the FOSs with the disturbance $(p \alpha<(p+1) \alpha)$. In addition, for those disturbances with small high order component, our method still works well. One application with the most potential is the realization of disturbance observance-based control (DOBC), which means that one can use the proposed observance method to estimate the external disturbance and the uncertainty and then achieve the desired response by an appropriate control.

\section{Illustrative Examples}

Example 1. Consider the system [27] as follows:

$$
\mathscr{D}^{0.8} x(t)=f(x, u ; t)+F d(t), \quad x(0)=4,
$$




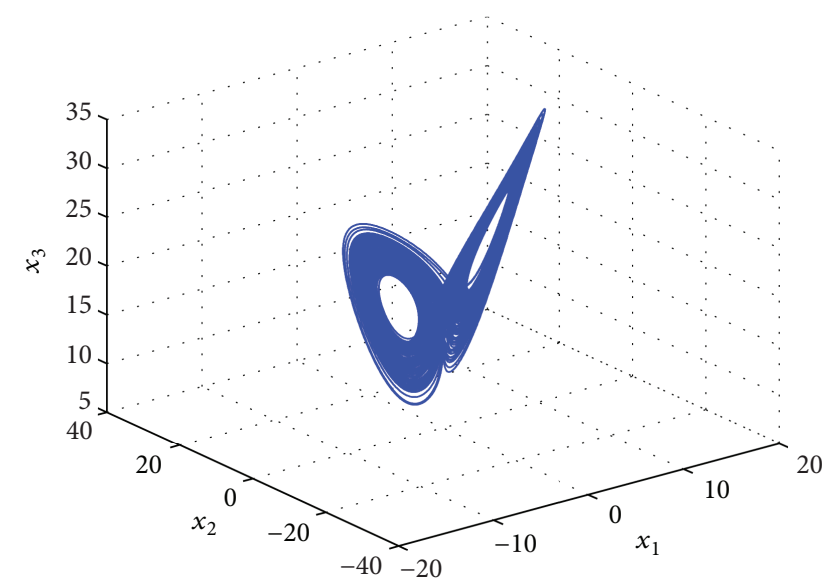

FIGURE 1: Chaotic attractor of the system with $d(t)=0$.

where $d(t)$ is a square wave and $f(x, u ; t), F$, and $x(0)$ satisfy

$$
\begin{gathered}
f(\cdot)=\left[\begin{array}{c}
-35 x_{1}(t)+35 x_{2}(t)+u_{1}(t) \\
-7 x_{1}(t)+28 x_{2}(t)-x_{1}(t) x_{3}(t)+u_{2}(t) \\
-3 x_{1}(t)+x_{1}(t) x_{2}(t)+u_{3}(t)
\end{array}\right], \\
F=\left[\begin{array}{lll}
1 & 1 & 1
\end{array}\right]^{T}, \quad x(0)=\left[\begin{array}{lll}
6 & 9 & 15
\end{array}\right]^{T} .
\end{gathered}
$$

We design the observer FODB0 as follows:

$$
\begin{gathered}
\widehat{d}(t)=\lambda_{0} g_{0}\left(F^{+} x(t)-z(t)\right), \\
\mathscr{D}^{0.8} z(t)=F^{+} f(x, u ; t)+\frac{\widehat{d}}{t s(t)},
\end{gathered}
$$

where $z(0)=F^{+} x(0)$; the nonlinear weighted function $g_{0}(\cdot)$ is shown as in Theorem 2.

Simulations are performed for $d(t)=0 ; u(t)=0$. The results shown in Figure 1 indicate that the original fractional system is chaotic.

Set the numerical simulation parameters $\left(\lambda_{0}, \sigma, w, T_{0}\right)$ as $(*, 5,0.1,0.005),(5, *, 0.1,0.005),(20,5, *, 0.005)$, and $(20,5,001, *, 0.005)$, respectively; then one obtains Figures 2 , 3,4 , and 5 .

Figure 2 shows the performance of FDOB0 depending on the design parameter $\lambda_{0}$. As $\lambda_{0}$ increases, FDOB0 results in fast estimation for the disturbance. Figure 3 shows that when $\sigma$ is big, the disturbance observers perform dynamically fast, which also indicates that the nonlinear function can carried over advantages for FOSs. One can observe from Figure 4 that the disturbance estimation becomes faster and faster as $w$ decreases. Figure 5 shows that arranging the transient dynamic can avoid the possible observation overshoot and observation time will increase with the increase of $T_{0}$.

Example 2. Considering the system in (46) with a sawtooth disturbance $d(t)$, we design the disturbance observer based on the FDOB0 and the FDOB1 without control, respectively. Selecting the parameters $\sigma=5, w=0.3, T_{0}=0.005$, and $\lambda_{0}=20$ for FDOB0 and $\lambda_{0}=40$ and $\lambda_{1}=400$

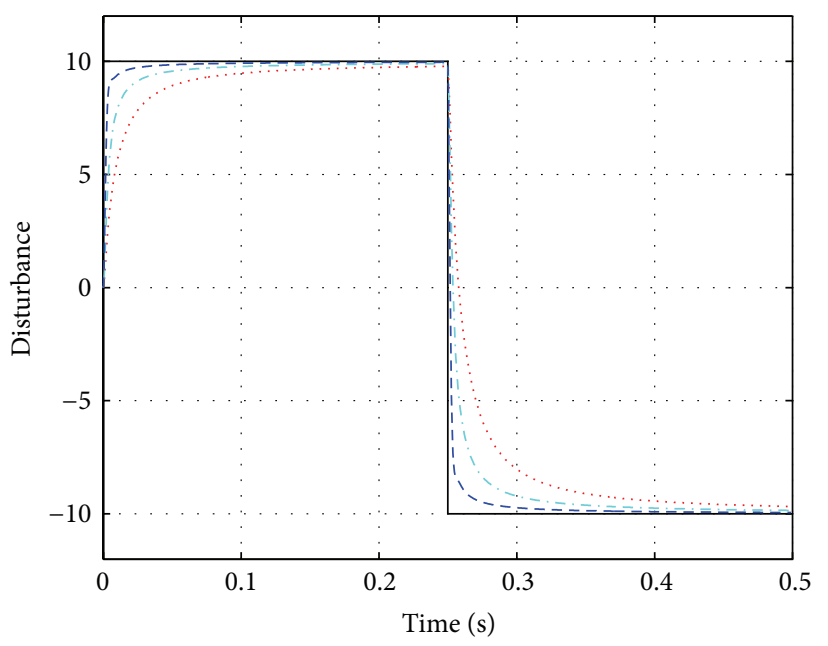

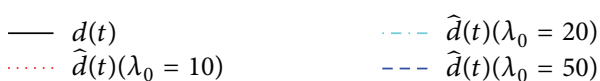

Figure 2: Results of Example 1 with different $\lambda_{0}$.

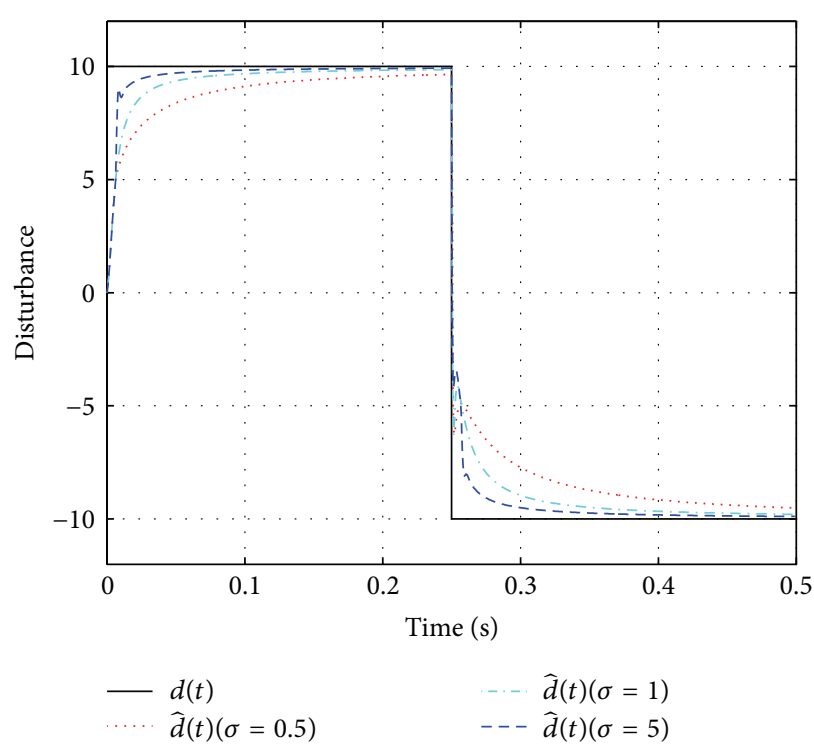

Figure 3: Results of Example 1 with different $\sigma$.

for FDOB1, then one gets the simulation results shown in Figure 6. Observe that FDOB1 can estimate the slope forms disturbance asymptotically, while the FDOB0 has the steady state error.

Example 3. Considering the same system in (46) with a higher order $n_{k}=2$ disturbance, we design FDOB0, FDOB1, and FDOB2 without control, respectively, by choosing the sets of parameters, $\lambda_{0}, \lambda_{1}$, and $\lambda_{2}$, such that $(10,0,0)$ for FDOB0, $(20,100,0)$ for FDOB1, and $(30,300,1000)$ for FDOB2. Other simulation parameters are selected as $\sigma=5$, $w=0.3$, and $T_{0}=0.005$. Note that the gain parameters are simply chosen to assign all the poles of the linear error dynamics at $s=(-10)^{1 / 0.8}$. As can be seen in Figure 7 , 


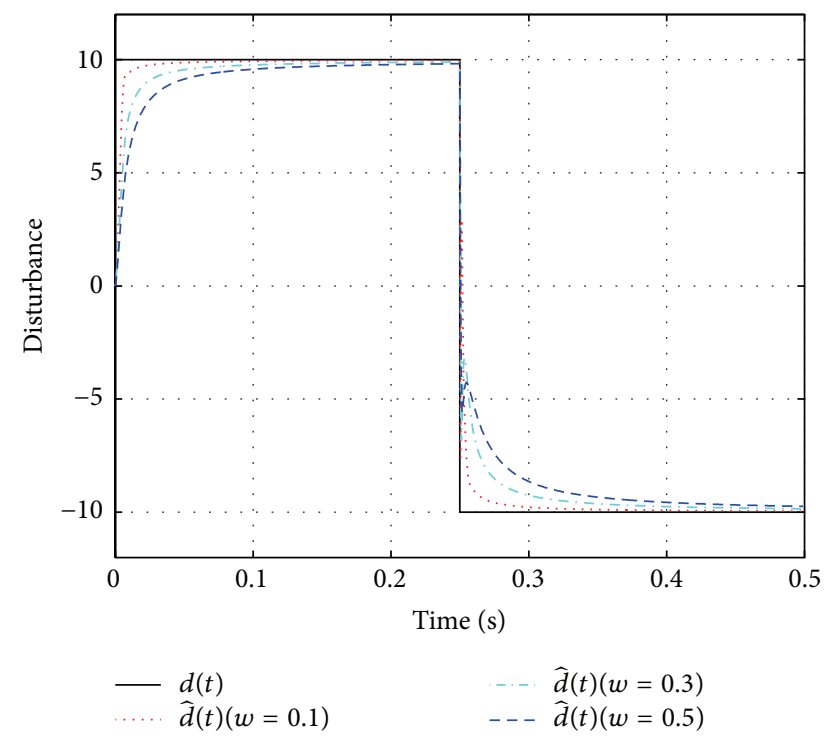

FIgURE 4: Results of Example 1 with different $w$.

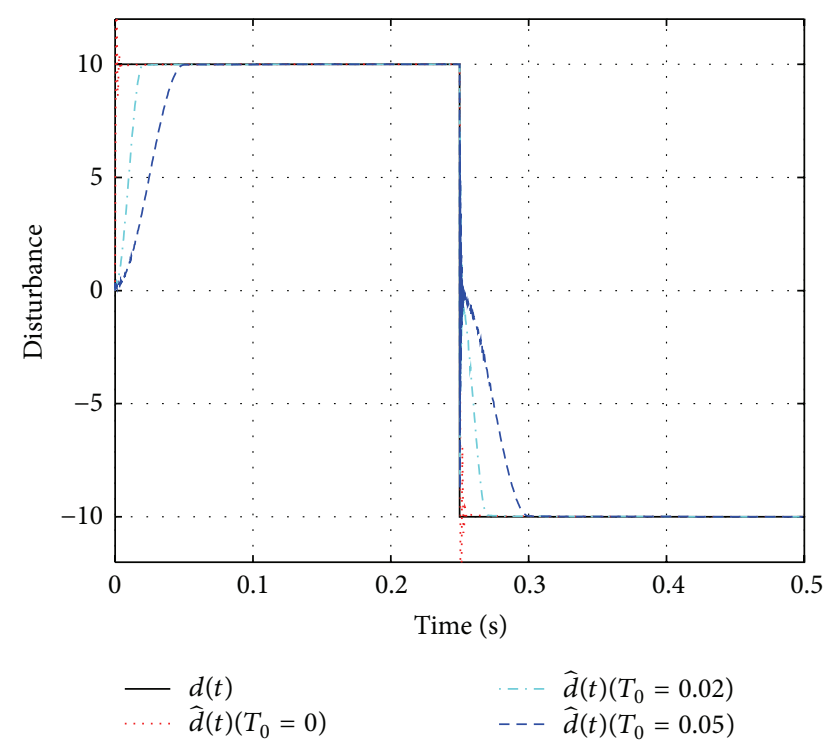

Figure 5: Results of Example 1 with different $T_{0}$.

the disturbance observer can be made dynamically fast by incorporating the higher order integrals.

Example 4. Considering the same system in (46) with a square wave disturbance, we design FDOB0 and state feedback controller, respectively, as follows:

$$
\begin{gathered}
\widehat{d}(t)=\lambda_{0} g_{0}\left(F^{+} x(t)-z(t)\right), \\
\mathscr{D}^{0.8} z(t)=F^{+} f(x, u ; t)+\frac{\widehat{d}}{t s(t)}, \\
u(t)=K x(t)-F \widehat{d}(t),
\end{gathered}
$$

where $K=\operatorname{diag}(-10,-51,-13)$.

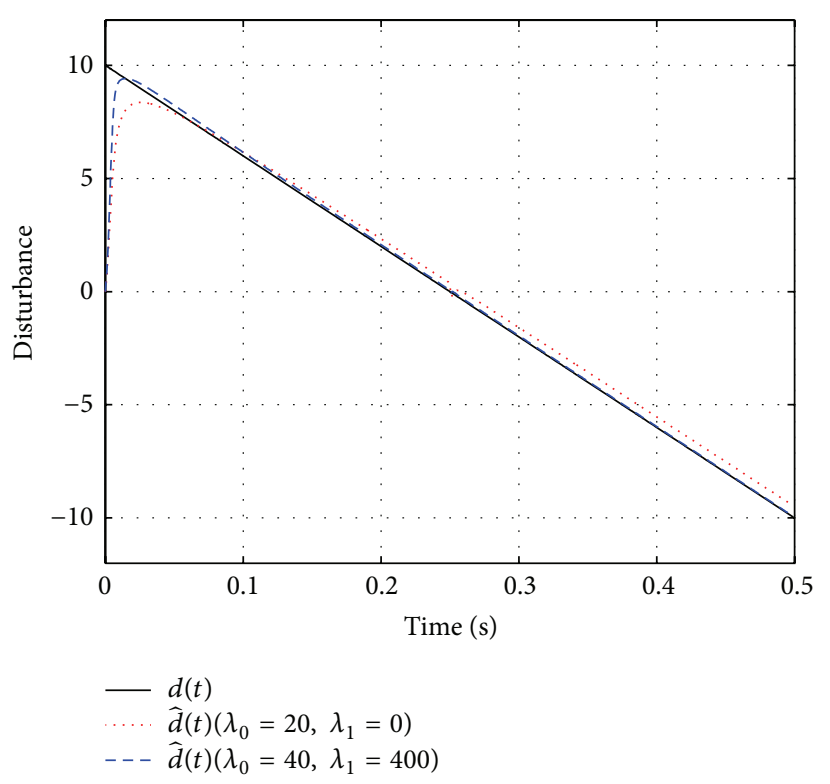

FIgURE 6: Results of Example 2 with FDOB0 and FDOB1.

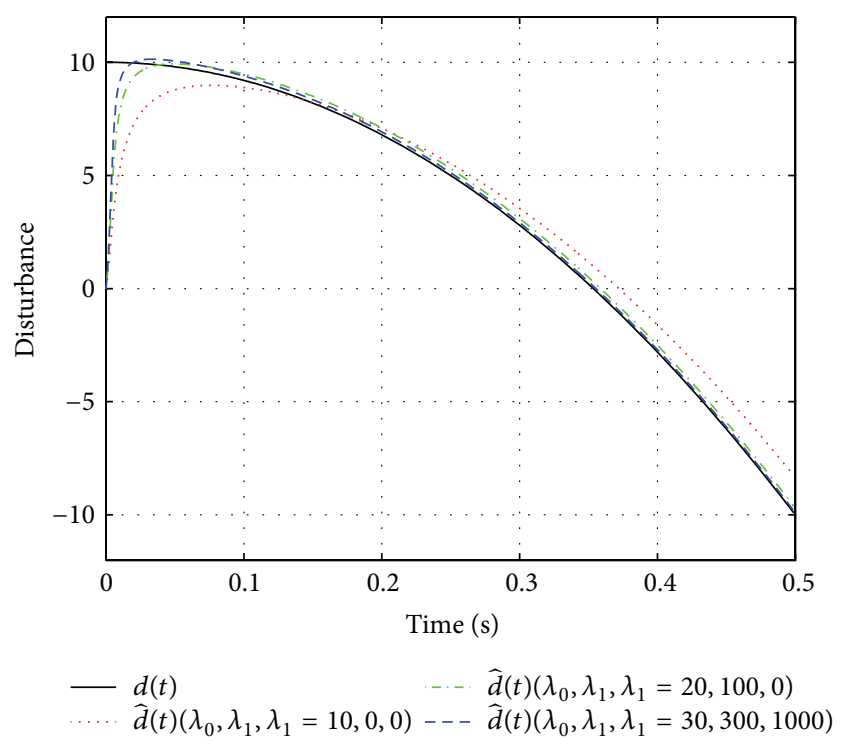

FIgURE 7: Results of Example 3 with FDOB0, FDOB1, and FDOB2.

This simulation is implemented with $\sigma=5, w=0.3$, and $T_{0}=0.005$. The related results are shown in Figure 8, from which one can observe that the state feedback controller based on FDOB0 can stabilize the original nonlinear system well. Also, it explicitly shows that the FDOB0 plays a good role in observing and eliminating the system disturbance. Combined with the nominal outer-loop controller, the FDOB0 can be used as an inner-loop compensator to control the FOSs with such disturbance.

\section{Conclusions}

In this paper, the methods of observer for fractional order systems in time series expansion disturbance have been 

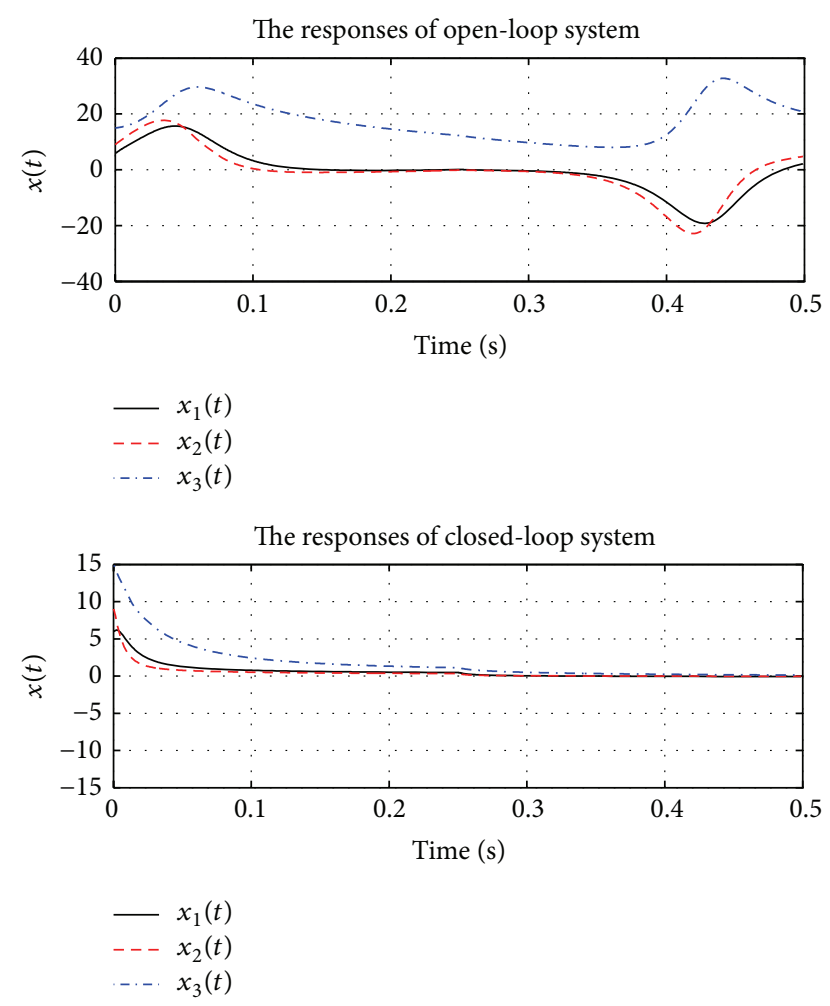

FIGURE 8: Results of Example 4 with control or not.

investigated. According to the maximum degree of disturbance polynomials, the disturbances are divided into three categories. And then, different observers have been designed for three disturbances, respectively. Compared to existing integer order results, the new proposed approaches have greater design freedom and the designed observers have a faster convergent speed. The numerical examples have shown the advantages and the efficiency of the proposed design methods. It is believed that the approaches provide a new avenue to solve such problems. The interesting future topics involve the following cases:

(i) to study the problem of noise effect reduction in case the measured state is mixed with the measurement noise;

(ii) to discuss the problem of output-based method when only partial states are measurable;

(iii) to investigate the problem considering the missing measurement data.

\section{Conflict of Interests}

The authors declare that there is no conflict of interests regarding the publication of this paper.

\section{Acknowledgments}

The authors would like to thank the associate editor and the anonymous reviewers for their keen and insightful comments which greatly improved the contents and the presentation. This work is supported by the National Nature Science Foundation under Grant no. 61004017.

\section{References}

[1] C. A. Monje, Y. Q. Chen, B. M. Vinagre, D. Xue, and V. Feliu, Fractional-Order Systems and Controls: Fundamentals and Applications, Advances in Industrial Control, Springer, London, UK, 2010.

[2] A. M. Lopes and J. A. Tenreiro Machado, "Root locus practical sketching rules for fractional-order systems," Abstract and Applied Analysis, vol. 2013, Article ID 102068, 14 pages, 2013.

[3] Z. Liao, Z. T. Zhu, S. Liang, C. Peng, and Y. Wang, "Subspace identification for fractional order hammerstein systems based on instrumental variables," International Journal of Control, Automation, and Systems, vol. 10, no. 5, pp. 947-953, 2012.

[4] S. Liang, C. Peng, Z. Liao, and Y. Wang, "State space approximation for general fractional order dynamic systems," International Journal of System Science, 2013.

[5] Z. Liao, C. Peng, W. Li, and Y. Wang, "Robust stability analysis for a class of fractional order systems with uncertain parameters," Journal of the Franklin Institute, vol. 348, no. 6, pp. 11011113, 2011.

[6] R. Sakthivel, P. Revathi, and N. I. Mahmudov, "Asymptotic stability of fractional stochastic neutral differential equations with infinite delays," Abstract and Applied Analysis, vol. 2013, Article ID 769257, 9 pages, 2013.

[7] S. Liang, C. Peng, and Y. Wang, "Improved linear matrix inequalities stability criteria for fractional order systems and robust stabilization synthesis: the $0<\alpha<1$ case," Control Theory \& Applications, vol. 30, no. 4, pp. 531-535, 2013.

[8] C. L. Li, K. L. Su, J. Zhang, and D. Q. Wei, "Robust control for fractional-order four-wing hyperchaotic system using LMI," Optik-International Journal for Light and Electron Optics, vol. 124, no. 22, pp. 5807-5810, 2013.

[9] M. M. Asheghan, S. S. Delshad, M. T. Hamidi Beheshti, and M. S. Tavazoei, "Non-fragile control and synchronization of a new fractional order chaotic system," Applied Mathematics and Computation, vol. 222, pp. 712-721, 2013.

[10] J. Yuan, B. Shi, and W. Ji, "Adaptive sliding mode control of a novel class of fractional chaotic systems," Advances in Mathematical Physics, vol. 2013, Article ID 576709, 13 pages, 2013.

[11] Y. Q. Chen, B. M. Vinagre, and I. Podlubny, "Fractional order disturbance observer for robust vibration suppression," Nonlinear Dynamics, vol. 38, no. 1-4, pp. 355-367, 2004.

[12] W. Li and Y. Hori, "Vibration suppression using single neuronbased PI fuzzy controller and fractional-order disturbance observer," IEEE Transactions on Industrial Electronics, vol. 54, no. 1, pp. 117-126, 2007.

[13] L. E. Olivier, I. K. Craig, and Y. Q. Chen, "Fractional order and BICO disturbance observers for a run-of-mine ore milling circuit," Journal of Process Control, vol. 22, no. 1, pp. 3-10, 2012.

[14] J. Q. Han, "From PID to active disturbance rejection control," IEEE Transactions on Industrial Electronics, vol. 56, no. 3, pp. 900-906, 2009.

[15] C. Qin, N. Qi, R. Lü, and K. Zhu, "ADRC fractional order PID controller design of hypersonic flight vehicle," Transactions of Nanjing University of Aeronautics \& Astronautics, vol. 28, no. 3, pp. $240-245,2011$. 
[16] M. D. Li, D. H. Li, J. Wang, and C. Zhao, "Active disturbance rejection control for fractional-order system," ISA Transactions, vol. 52, no. 3, pp. 365-374, 2013.

[17] K. Erenturk, "Fractional-order $\mathrm{PI}^{\lambda} \mathrm{D}^{\mu}$ and active disturbance rejection control of nonlinear two mass drive system," IEEE Transactions on Industrial Electronics, vol. 60, no. 9, pp. 38063813, 2013.

[18] J. Hu, Z. Wang, B. Shen, and H. Gao, "Quantised recursive filtering for a class of nonlinear systems with multiplicative noises and missing measurements," International Journal of Control, vol. 86, no. 4, pp. 650-663, 2013.

[19] J. Hu, Z. Wang, and H. Gao, "Recursive filtering with random parameter matrices, multiple fading measurements and correlated noises," Automatica, vol. 49, no. 11, pp. 3440-3448, 2013.

[20] J. Hu, Z. Wang, B. Shen, and H. Gao, "Gain-constrained recursive filtering with stochastic nonlinearities and probabilistic sensor delays," IEEE Transactions on Signal Processing, vol. 61, no. 5, pp. 1230-1238, 2013.

[21] Z. Xiang and R. Wang, "Non-fragile observer design for nonlinear switched systems with time delay," International Journal of Intelligent Computing and Cybernetics, vol. 2, no. 1, pp. 175-189, 2009.

[22] A. Pisano, M. Rapai'c, and E. Usai, Second-Order Sliding Mode Approaches to Control and Estimation for Fractional Order Dynamics, Springer, Berlin, Germany, 2012.

[23] M. Xiang and Z. Xiang, "Observer design of switched positive systems with time-varying delays," Circuits, Systems, and Signal Processing, vol. 32, no. 5, pp. 2171-2184, 2013.

[24] G. Fedele and A. Ferrise, "Periodic disturbance rejection with unknown frequency and unknown plant structure," Journal of the Franklin Institute, vol. 351, no. 2, pp. 1074-1092, 2014.

[25] X. Li and Z. Xiang, "Observer design of discrete-time impulsive switched nonlinear systems with time-varying delays," Applied Mathematics and Computation, vol. 229, pp. 327-339, 2014.

[26] K. S. Kim, K. H. Rew, and S. Kim, "Disturbance observer for estimating higher order disturbances in time series expansion," IEEE Transactions on Automatic Control, vol. 55, no. 8, pp. 19051911, 2010.

[27] S. K. Agrawal and S. Das, "A modified adaptive control method for synchronization of some fractional chaotic systems with unknown parameters," Nonlinear Dynamics, vol. 73, no. 1-2, pp. 907-919, 2013. 


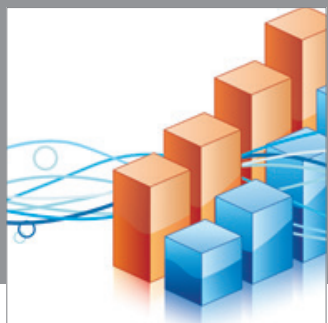

Advances in

Operations Research

mansans

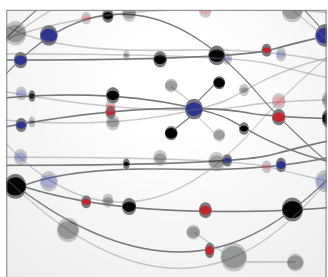

The Scientific World Journal
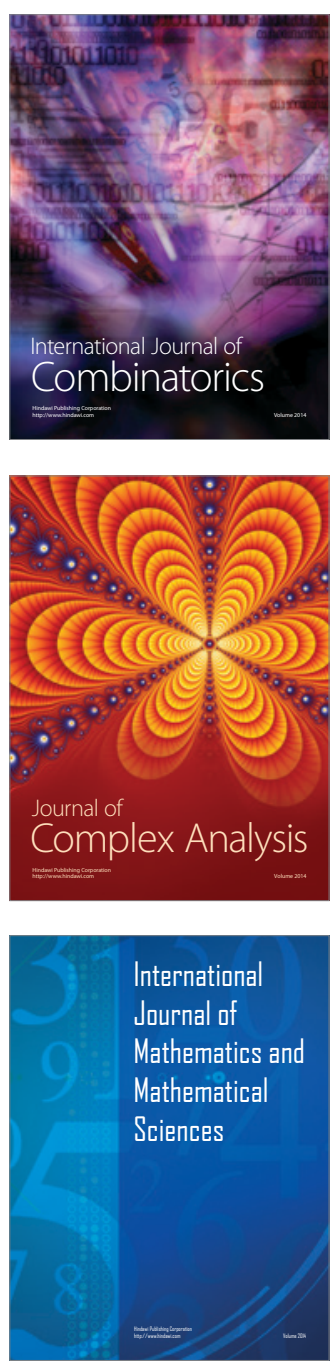
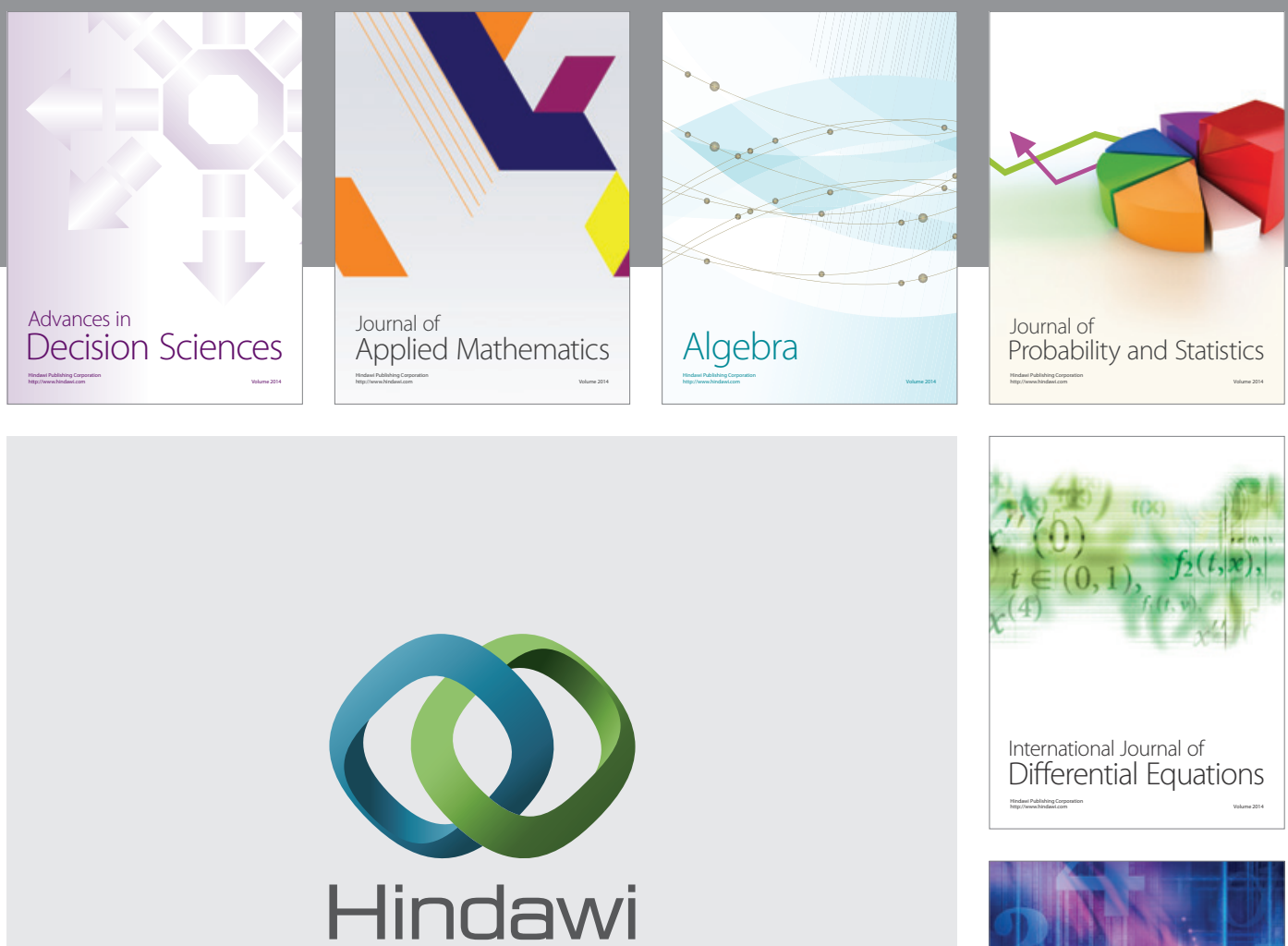

Submit your manuscripts at http://www.hindawi.com
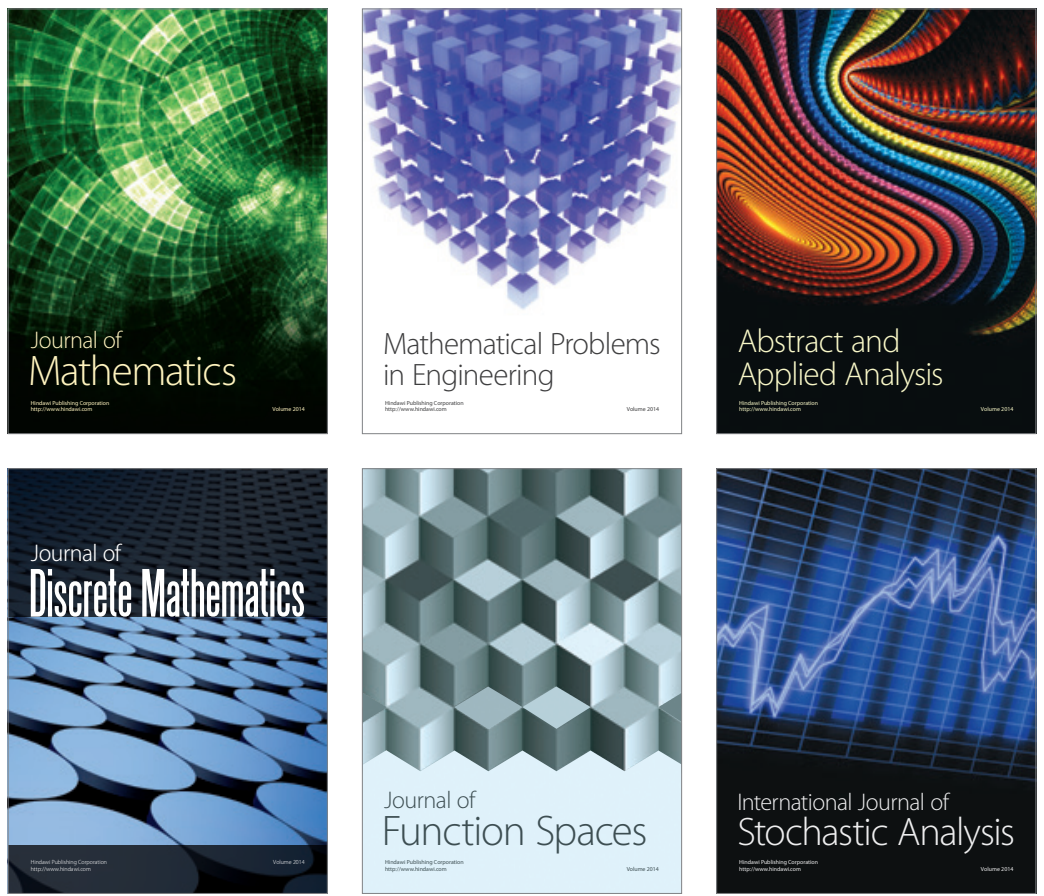

Journal of

Function Spaces

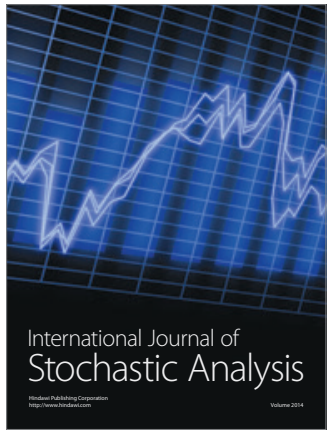

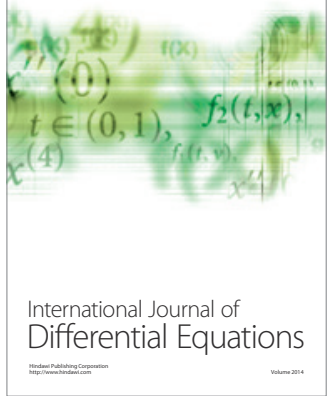
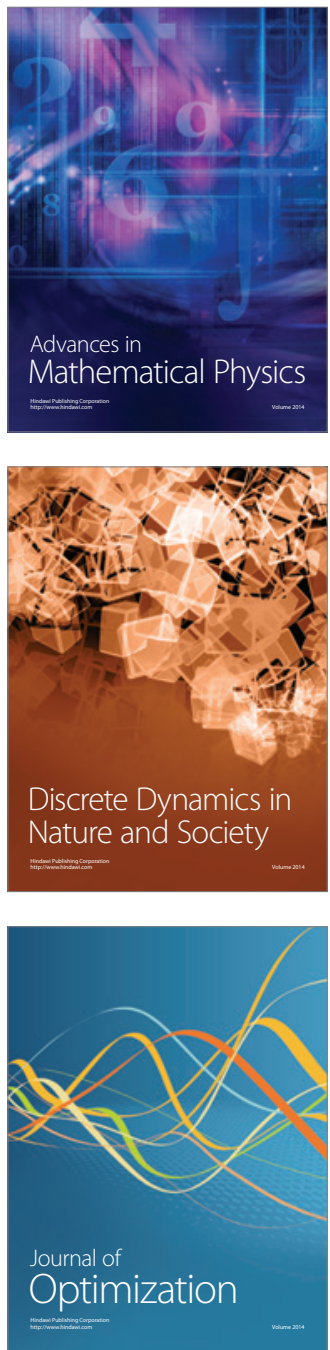